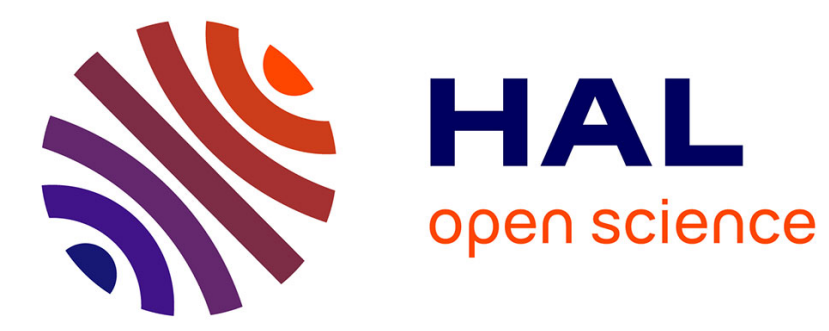

\title{
Le contrôle de gestion des clubs de football professionnel
}

François Meyssonnier, Myriam Mincheneau

\section{- To cite this version:}

François Meyssonnier, Myriam Mincheneau. Le contrôle de gestion des clubs de football professionnel. 2014. hal-00936874

\section{HAL Id: hal-00936874 https://hal.science/hal-00936874}

Preprint submitted on 27 Jan 2014

HAL is a multi-disciplinary open access archive for the deposit and dissemination of scientific research documents, whether they are published or not. The documents may come from teaching and research institutions in France or abroad, or from public or private research centers.
L'archive ouverte pluridisciplinaire HAL, est destinée au dépôt et à la diffusion de documents scientifiques de niveau recherche, publiés ou non, émanant des établissements d'enseignement et de recherche français ou étrangers, des laboratoires publics ou privés. 
EA 4272

\title{
Le contrôle de gestion des clubs de football professionnel
}

\author{
François Meyssonnier* \\ Myriam Mincheneau**
}

2014/06

$\left(^{*}\right)$ LEMNA - Université de Nantes

${ }^{* *}$ School of Management, Audencia Nantes

\section{Laboratoire d'Economie et de Management Nantes-Atlantique} Université de Nantes

Chemin de la Censive du Tertre - BP 52231

44322 Nantes cedex 3 - France

www.univ-nantes.fr/iemn-iae/recherche

Tél. +33 (0)2 40141717 - Fax +33 (0)2 40141749 


\title{
Le contrôle de gestion des clubs de football professionnel
}

\author{
Management Control of Professional Football Clubs \\ JEL classification : M 40 (Accounting and Auditing - General)
}

\section{Résumé :}

La nature et les modalités de l'activité des clubs de football professionnel sont étudiées et des entretiens avec un panel d'experts sont réalisés. Une analyse des pratiques et outils de contrôle de gestion de huit clubs représentatifs des quarante des ligues 1 et 2 de football est présentée. Ceci permet de comprendre et d'expliquer les modes de structuration des systèmes de pilotage de la performance économique dans le sport spectacle.

Mots-clés : sport spectacle, clubs de football professionnel, pilotage de la performance économique, outils du contrôle de gestion.

\begin{abstract}
:
Nature and modalities of the activity of professional football clubs are studied and interviews with a panel of experts are realized. An analysis of management control practices and tools of eight clubs representatives of the forty of football leagues 1 and 2 is presented. This allows to understand and to explain the structuring modes of the economic performance management systems in sport entertainment.

Keywords: sport entertainment, professional football clubs, economic performance management, tools of management control.
\end{abstract}




\section{Introduction}

Une nouvelle économie se déploie actuellement, fondée sur la globalisation des marchés et des acteurs et intégrant l'impact profond et structurant des nouvelles technologies de la communication sur les comportements des consommateurs et des entreprises. Dans ce contexte, de nouvelles activités émergent et jouent un rôle de plus en plus important à côté des activités industrielles classiques : ce sont par exemple, les industries innovantes basées sur la science (high tech start-up) ou les activités de divertissement (entertainment business). Nous allons nous intéresser ici au sport spectacle qui relève de la deuxième catégorie et qui est susceptible de nous apporter beaucoup dans la compréhension des démarches et outils du contrôle de gestion dans un contexte où la finalité est complexe (recherche conjointe du résultat sportif et du résultat financier), la prestation de service est coproduite avec un concurrent (le match entre deux équipes comme spectacle), la gouvernance est difficile (faible maitrise des actifs immatériels principaux que constituent les joueurs) et le risque est inhérent à l'activité (l'incertitude du résultat sportif en fait son attrait pour le spectateur).

La vision classique du contrôle de gestion admise depuis Anthony (1965) le réduit trop souvent aux techniques universelles fondées sur la modélisation financière telles que la comptabilité de gestion et le système budgétaire et à la séparation des rôles de contrôle entre les contrôleurs de gestion et les managers opérationnels. Même si plus tard Anthony et Govindarajan (1995) ajoutent au champ du contrôle le contrôle stratégique, cette démarche est régulièrement remise en cause. Une approche rénovée et élargie, centrée sur les processus opérationnels et considérant que le contrôle de gestion doit prendre en compte l'ensemble des dispositifs qui assurent la déclinaison efficace et efficiente du business model jusqu'au niveau opérationnel, est développée maintenant par plusieurs auteurs (Giard et Lagroue 2001 ; Malmi et Brown 2008 ; Ferreira et Otley 2009 ; Meyssonnier 2013). En insistant sur les processus opérationnels de terrain, une large place est faite alors à l'approche par le métier et l'analyse est contingente et non plus universelle.

C'est dans le cadre d'une telle démarche fondée sur une vision à la fois plus large du contrôle de gestion mais aussi plus contextualisée, que nous avons fait le choix d'étudier les modes de structuration des systèmes de pilotage de la performance économique du secteur 
probablement le plus développé du sport spectacle en France, le football professionnel. Il s'agit pour nous d'approfondir la compréhension empirique des dispositifs de contrôle de gestion mis en œuvre dans les clubs français de football professionnel. Pour cela, nous définirons d'abord la nature, le contexte et les spécificités de la gestion des clubs (partie 1). Nous exposerons ensuite la méthodologie et les terrains de notre recherche (partie 2). Nous présenterons les résultats de l'étude (partie 3). Nous pourrons enfin discuter ces résultats (partie 4).

\section{Le contexte de gestion des clubs français de football professionnel}

Les caractéristiques du football professionnel vont être présentées puis une typologie des clubs va être proposée.

\subsection{Performance sportive et/ou performance économique}

Dans certains pays, le sport spectacle est un business comme un autre et les acteurs économiques y investissent afin de créer de la valeur financière. C'est, par exemple, le cas en Amérique du Nord où les principales compétitions sportives sont organisées en ligues fermées ce qui évite le spectre de la relégation et ses conséquences financières. En France, les quarante clubs de football de ligue 1 et de ligue 2 ont le statut de sociétés commerciales. Mais, si formellement, le cadre juridique est tout à fait comparable à celui des entreprises privées classiques des autres secteurs de l'économie, la réalité économique actuelle des clubs est assez différente.

Selon la DNCG, l'ensemble des clubs de L1 et L2 a généré un CA hors mutation des joueurs de 1,3 milliard d' $€$ en 2011/12, réparti en droits audiovisuels (53\%), en produits liés aux sponsors et publicité (17\%), en recettes de matchs (12\%) et en produits dérivés et subventions (18\%). La rémunération globale du personnel, composée principalement des joueurs, représente $75 \%$ des charges d'exploitation des clubs et le déficit d'exploitation cumulé des 40 clubs est de plus de $323 \mathrm{M} €$. Les produits apparaissent très dépendants de la performance sportive donc fortement aléatoires tandis que les charges sont principalement fixes. Selon Dermit-Richard (2003), les clubs sont malades de leurs coûts car aucun gain de productivité n'a pu être réalisé par les clubs alors que les salaires des joueurs ont littéralement explosé depuis l'arrêt Bosman en 1995. Ainsi, cette activité est depuis des décennies structurellement 
déficitaires et lorsqu'un bénéfice apparaît, il sert souvent à combler des déficits antérieurs ou est mis en réserve pour anticiper un éventuel déficit ultérieur (Rousseau 2009 ; Leroux 2011).

Peu de propriétaires acceptent aujourd'hui de combler continuellement les déficits des clubs et la régulation mise en place par la DNCG a pour objectif l'équilibre financier. La performance sportive est prédominante mais la dimension financière n'est pas une simple variable d'ajustement: l'objectif d'un club est en général d'optimiser des résultats sportifs sous contrainte d'équilibre des comptes (Kesenne 2002). La première caractéristique de la gestion des clubs de football professionnel est donc cette complexité de leur finalisation.

\subsection{Une gouvernance partenariale}

$\mathrm{Au}$ sein des clubs, diverses parties prenantes évoluent avec des degrés d'influence différents (Senaux 2004) et l'économie du football professionnel tourne autour de la valorisation de trois actifs immatériels (Paché et N'Goala 2011) : le «capital humain » des joueurs (effectif et talent des joueurs du club); le capital «marque » du club (notoriété et image du club); le capital «clients» des spectateurs et des téléspectateurs (valeur actualisée des revenus à venir provenant des supporters et des abonnés, des diffuseurs et des sponsors).

Le marché des joueurs est dual. Le marché primaire est composé de joueurs de haut niveau international à rémunérations très élevées, mobiles internationalement, ayant même parfois une valeur économique supérieure à leur valeur sportive (ex : Beckam lors de son passage au PSG) et le marché secondaire est composé de joueurs interchangeables moins rémunérés (Gouguet et Primault 2002). L'internationalisation du recrutement des joueurs et l'importance accrue des agents sportifs et des intermédiaires ont eu comme conséquence «l'augmentation massive des salaires, la réduction de la durée effective des contrats et l'augmentation du nombre de transferts entre les clubs »(Brocard 2009). Face à des dépenses salariales incompressibles à court terme, les clubs cherchent à développer des recettes stables et pérennes (droits TV, produits dérivés, activités liées, meilleure utilisation du stade...) mais cela n'est possible que si le club arrive à constituer une marque identifiée (Couvaelere et Richelieu 2005) et valorisée au niveau européen avec des joueurs internationalement connus, ce qui n'est le cas que de peu de clubs (Paris, Lyon, Marseille). Les clients des clubs (supporters, abonnés) 
représentent la dernière source de valeur dans l'économie du football professionnel. Les clubs utilisent tous les outils du marketing expérientiel pour développer les recettes liées au merchandising en jouant sur l'attachement sentimental du supporter au club ou à son identification à un joueur vedette et en essayant de faire en sorte que le stade devienne un lieu de vie (Fontanel 2007).

A côté des parties prenantes principales (actionnaires, joueurs, spectateurs) dont l'implication dans la vie du club est nécessaire à un degré ou un autre à la réussite du club, on peut identifier des parties prenantes secondaires : les instances dirigeantes (l'UEFA, la Fédération Française de Football et de la Ligue de Football Professionnel); les collectivités locales; l'association support et le monde amateur. Leurs influences sont particulièrement significatives dans le cas des petits clubs. La ligue professionnelle leur garantit la majorité de leurs revenus (droits TV) et leur impose un minimum de règles de gestion par le biais de la DNCG. Les collectivités locales aident les clubs (le plus souvent en investissant dans des équipements sportifs, en achetant de prestations de services et en subventionnant la formation) ce qui est crucial surtout pour les clubs ne pouvant s'appuyer sur un tissu économique important (aires urbaines réduites). Enfin, le monde amateur est un acteur local de poids pour les plus petits clubs de L2.

Il faut toutefois aborder cette notion de gouvernance partenariale de façon prudente car elle fait référence à des rapports de force et à des influences croisées. Les aspects individuels et particuliers sont prédominants. Les flux financiers illégaux de l'industrie du sport, liés au dopage et à la corruption en faveur d'une des deux équipes ou dans le cas de paris sportifs, se sont aussi développés (Drut 2011). Ils seraient liés principalement au blanchiment d'argent (FATF-GAFI 2009) même si les paris légaux contribuent au financement du sport professionnel en France depuis mai 2010. D'autres dérives financières prennent aussi de l'ampleur à cause de la volonté de certains de contourner les régulations mises en place dans leur pays : prêts fictifs aux joueurs et paiement de contrats d'image aux sportifs via des sociétés situées dans des paradis fiscaux, abus de biens sociaux, etc. (Andreff 2010; Bourg et Gouguet 2012). Ces évolutions traduisent une dépendance accrue des clubs français vis à vis de parties prenantes difficilement maîtrisables. 


\subsection{Une situation d'incertitude consubstantielle au spectacle sportif}

Il a été démontré statistiquement et sur le long terme qu'il existe un lien entre le budget et le potentiel économique local (Gouguet et Primault 2006 ; Helleu et Durand 2007) et une forte corrélation entre les budgets des clubs et le classement du championnat (Bourg et Gouguet 1998 ; Hoehn et Szymanski 1999). Pour la saison 2011/12, le coefficient de corrélation était de 0,89 pour la ligue 1 (DNCG 2012, p. 61). Pourtant, une partie de l'intérêt du sport spectacle réside dans l'incertitude du résultat. Le plaisir d'un spectateur dépendra de la qualité technique d'un match mais aussi et surtout du suspense sur le résultat. L'enjeu domine la qualité du jeu et fait que le spectacle sportif est attractif. La Ligue de Football Professionnel en redistribuant les droits TV contribue au maintien de petits clubs qui peuvent mettre en difficulté ponctuellement les grands au cours d'un championnat mais surtout dans les compétitions à élimination directe (coupes) et recréer ainsi des situations d'incertitude tout au long de compétitions attirantes pour les spectateurs et téléspectateurs. Le spectacle sportif est donc consubstantiellement lié au risque significatif que Touchais (2001) évalue au deuxième niveau de l'échelle de Courtney et al. (1997) composée de 4 niveaux. Les clubs ont la possibilité de prévoir plusieurs futurs à l'aide de probabilités de réalisation de chaque scénario, d'un modèle d'analyse de la décision, de la théorie des jeux ou de l'évaluation d'options. Fréry (2009) suggère six postures stratégiques et notamment d' «accepter l'imprévu» en choisissant d'imiter ses concurrents ou d'adopter une structure d'entreprise agile. Dans ce dernier cas, l'objectif est de « concevoir une organisation suffisamment adaptable, flexible et résiliente » grâce à l'externalisation d'une partie de sa chaîne de valeur et au transfert des risques à des prestataires externes (investissements financés par un tiers, contrats d'assurance, etc.). Un système d'animation basé sur la confiance en l'avenir peut également être créé en interne (Gervais 2009). Mais tant que les entreprises seront fondées sur une seule activité, ce qui est le cas des clubs, le degré d'incertitude restera important.

\subsection{Une classification indicative des clubs}

En prenant en compte les trois caractéristiques structurantes du football professionnel que nous venons d'identifier relativement à la finalisation, à la gouvernance et au risque, on peut établir une typologie 
des clubs. La DNCG analyse chaque année dans son rapport d'activité, les clubs en les regroupant selon l'importance des budgets. Cependant, au sein de chaque groupe, les clubs différent en fonction de leurs projets stratégiques, de leurs performances sportive et financière, de leur financement et de leur gouvernance. Ainsi, nous proposons de classer les clubs en 3 catégories: les grands clubs à vocation européenne, entreprises diversifiées de spectacle sportif avec une marque reconnue et un risque réduit par la capacité financière des investisseurs (Paris, Marseille, Lyon, Lille et Bordeaux); les clubs de dimension nationale cherchant à faire de leur stade un lieu de vie, à construire une histoire cohérente et à affirmer une marque au niveau national sur la durée (Montpellier, Nantes, Nice, Rennes, Saint-Etienne, Toulouse, etc.) ; les petits clubs régionaux qui doivent pérenniser par des résultats sportifs leur appartenance à la L2 en s'appuyant sur des parties prenantes secondaires comme les collectivités locales (les autres clubs de L2). Mais, il ne faut pas avoir une vision statique des clubs. Cette typologie est évolutive et on doit prendre en compte les trajectoires des clubs et les conséquences des aléas sportifs qu'ils subissent comme nous le montrons dans le schéma 1 ci-dessous.

Schéma 1: Contexte et modes de gestion des clubs de football professionnel

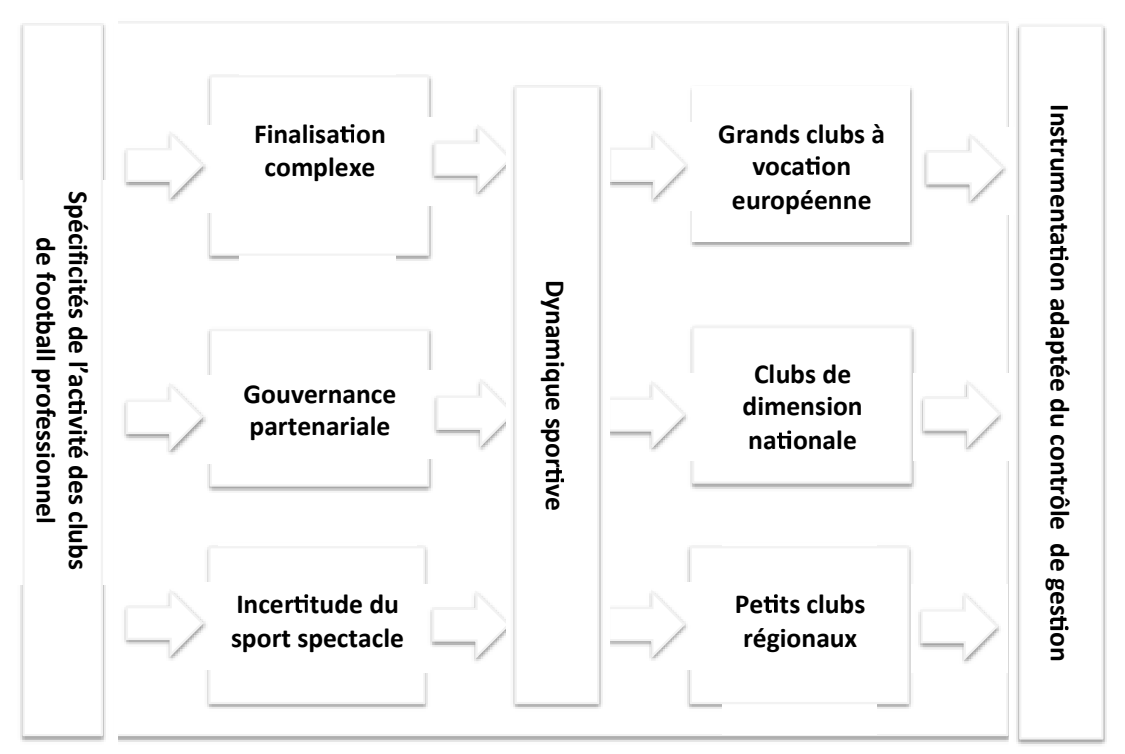


Après avoir décrit le contexte et les acteurs du football professionnel, nous allons présenter maintenant le design de notre recherche.

\section{La démarche de recherche}

Une phase exploratoire a été réalisée suivie d'une étude de terrain dans les clubs.

\subsection{Phase préliminaire}

Plusieurs recherches, mais assez anciennes, ont été déjà réalisées sur les pratiques et outils de gestion dans les clubs de football professionnel (Pelluault 1992; Augé 1998 ; Touchais 2001 ; Lauzanas 2003). Pour mieux explorer le degré de développement actuel des pratiques de contrôle de gestion dans le football professionnel, nous avons rencontrés, courant 2012, huit régulateurs ou spécialistes du domaine qui ont pu nous faire part de leurs points de vue sur la gestion des clubs de football professionnel (cf. tableau 1 ci-dessous).

Tableau $1:$ Les experts interviewés

\begin{tabular}{|c|c|c|c|}
\hline Nom & Qualité & Fonction & $\begin{array}{l}\text { Date de } \\
\text { l'entretien }\end{array}$ \\
\hline Mr A & Salarié de la Ligue de Football & Contrôleur de gestion & $\begin{array}{l}\text { Août } \\
2012\end{array}$ \\
\hline $\mathrm{Mr} \mathrm{B}$ & $\begin{array}{l}\text { Membre de la DNCG } \\
\text { et de l'UEFA }\end{array}$ & Expert-comptable & $\begin{array}{l}\text { Août } \\
2012\end{array}$ \\
\hline $\mathrm{MrC}$ & Membre de la DNCG & $\begin{array}{l}\text { Commissaire aux comptes } \\
\text { dans un des big four }\end{array}$ & $\begin{array}{l}\text { Septembre } \\
2012\end{array}$ \\
\hline Mr D & Membre de la DNCG & $\begin{array}{l}\text { Juriste au ministère } \\
\text { des sports }\end{array}$ & $\begin{array}{l}\text { Novembre } \\
2012\end{array}$ \\
\hline MmeE & $\begin{array}{l}\text { Salariée de l'Union des Clubs } \\
\text { Professionnels de Football }\end{array}$ & Juriste & $\begin{array}{l}\text { Septembre } \\
2012\end{array}$ \\
\hline $\mathrm{MrF}$ & Spécialiste du sport-spectacle & Consultant & $\begin{array}{l}\text { Septembre } \\
2012\end{array}$ \\
\hline $\mathrm{Mr} \mathrm{G}$ & $\begin{array}{l}\text { Spécialiste du contrôle } \\
\text { de gestion dans le sport }\end{array}$ & Universitaire & $\begin{array}{l}\text { Octobre } \\
2012\end{array}$ \\
\hline $\mathrm{MrH}$ & $\begin{array}{l}\text { Spécialiste des clubs sportifs } \\
\text { professionnels }\end{array}$ & $\begin{array}{l}\text { Commissaire aux comptes } \\
\text { dans un des big four }\end{array}$ & $\begin{array}{l}\text { Octobre } \\
2012\end{array}$ \\
\hline
\end{tabular}




\subsection{Etude des pratiques de contrôle de gestion dans huit clubs représentatifs}

Une enquête a ensuite été réalisée sur le terrain pour approfondir l'appréciation des experts et l'enrichir de l'étude de cas représentatifs de la diversité des situations en matière de contrôle de gestion dans les clubs de football professionnel.

D'octobre 2012 à mai 2013, des entretiens semi-directifs d'environ deux heures ont été réalisés avec une ou deux personnes (président, DG, DAF ou contrôleur de gestion) au sein de huit clubs. Des informations préparatoires avaient été recueillies au préalable et beaucoup de documentation a été collectée sur place. Un guide de conduite des entretiens semi-directifs avait été établi permettant d'aborder successivement les questions portant sur : la description du club et de son histoire; la présentation du business model, du fonctionnement et de la structure du club; la description des pratiques et outils de calcul de coûts, de budgétisation, d'usage d'indicateurs d'activité et de performance; le diagnostic et les perspectives des acteurs du terrain en matière de système de pilotage de la performance économique du club.

Le nombre de clubs rencontrés a été limité à huit en raison de l'application du principe de saturation dans l'accumulation de l'information qualitative de terrain: les derniers entretiens ne nous apportaient plus qu'une connaissance marginale assez faible. De plus, avec huit clubs étudiés sur les quarante des ligues 1 et 2, nous avons un échantillon de $20 \%$ de la population des clubs qui nous a semblé suffisamment représentatif. Il se compose ainsi, de :

- deux grands clubs à vocation européenne (GCVE) : $\mathrm{C} 1$ et $\mathrm{C} 2$;

- quatre clubs de dimension nationale (CDN) : C3, C4, C5, C6 ;

- deux petits clubs régionaux (PCR) : $\mathrm{C} 7$ et $\mathrm{C} 8$.

Nous avons laissé de côté de façon délibérée les trois clubs de L1 dont la nature est assez particulière: le PSG (en raison des capacités financières semble-t-il illimitées du propriétaire qatari), l'OL (club côté en bourse) et l'OM (en raison de la «complexité » de son mode de fonctionnement et de son environnement local). Toutes les indications relatives à ces clubs, qui ont souhaité rester anonymes, sont synthétisées dans le tableau 2 ci-après. 
Tableau 2: Les huit clubs étudiés

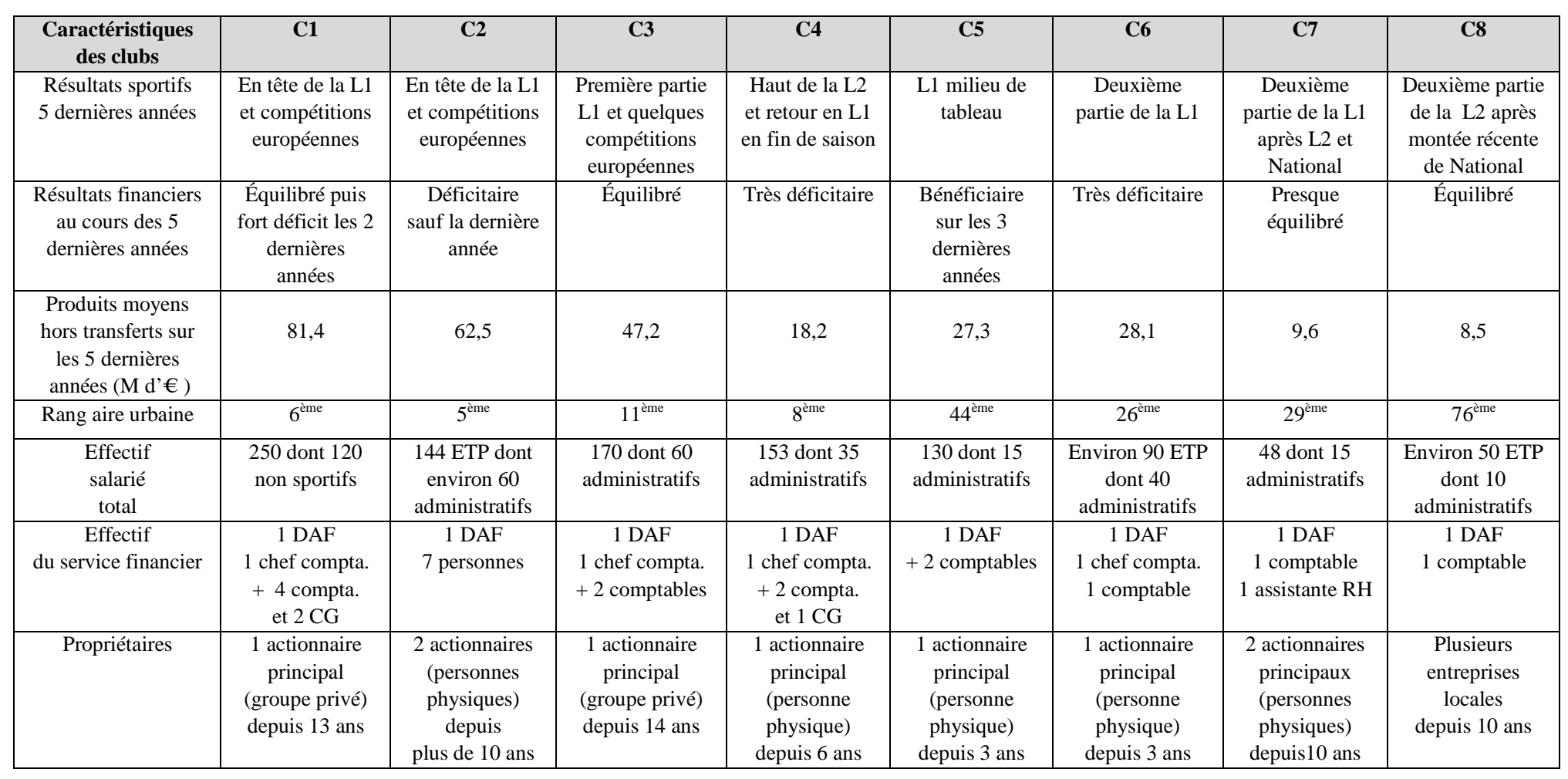




\begin{tabular}{|c|c|c|c|c|c|c|c|c|}
\hline Structure juridique & $\begin{array}{c}\text { SASP } \\
+3 \text { filiales à } \\
100 \% \\
+ \text { filiale } \\
\text { de formation }\end{array}$ & $\begin{array}{c}\text { SASP } \\
+3 \text { filiales à } \\
100 \% \\
+1 \text { à } 51 \% \\
+ \text { association } \\
\quad \text { pour la } \\
\text { formation }\end{array}$ & $\begin{array}{c}\text { SASP } \\
+ \text { 1filiale à } \\
100 \% \\
+ \text { filiale } \\
\text { formation }\end{array}$ & $\begin{array}{c}\text { SASP } \\
\text { intégrant la } \\
\text { formation }\end{array}$ & $\begin{array}{c}\text { SASP } \\
+2 \text { filiales à } \\
100 \% \\
+ \text { filiale } \\
\text { formation }\end{array}$ & $\begin{array}{c}\text { SASP } \\
\text { intégrant la } \\
\text { formation }\end{array}$ & $\begin{array}{c}\text { SASP } \\
\text { avec formation } \\
\text { gérée par } \\
\text { association }\end{array}$ & $\begin{array}{c}\text { SASP } \\
\text { intégrant la } \\
\text { formation }\end{array}$ \\
\hline $\begin{array}{c}\text { Présentation des } \\
\text { comptes }\end{array}$ & $\begin{array}{l}\text { DNCG } \\
\text { PCG } \\
\text { IFRS } \\
\text { UEFA }\end{array}$ & $\begin{array}{c}\text { DNCG } \\
\text { PCG } \\
\text { IFRS } \\
\text { UEFA }\end{array}$ & $\begin{array}{l}\text { DNCG } \\
\text { PCG } \\
\text { IFRS }\end{array}$ & $\begin{array}{l}\text { DNCG } \\
\text { PCG }\end{array}$ & $\begin{array}{l}\text { DNCG } \\
\text { PCG }\end{array}$ & $\begin{array}{c}\text { DNCG } \\
\text { PCG }\end{array}$ & $\begin{array}{c}\text { DNCG } \\
\text { PCG }\end{array}$ & $\begin{array}{c}\text { DNCG } \\
\text { PCG }\end{array}$ \\
\hline $\begin{array}{c}\text { Outils du système } \\
\text { d'information }\end{array}$ & $\begin{array}{l}\text { Progiciel } \\
\text { Tableur } \\
\text { CRM } \\
\text { Requêteur }\end{array}$ & $\begin{array}{c}\text { Progiciel } \\
\text { Tableur } \\
\text { CRM }\end{array}$ & $\begin{array}{c}\text { Progiciel } \\
\text { Tableur } \\
\text { CRM }\end{array}$ & $\begin{array}{c}\text { Progiciel } \\
\text { Tableur } \\
\text { CRM } \\
\text { Requêteur }\end{array}$ & $\begin{array}{c}\text { Progiciel } \\
\text { Tableur } \\
\text { CRM }\end{array}$ & $\begin{array}{c}\text { Progiciel } \\
\text { Tableur } \\
\text { CRM }\end{array}$ & $\begin{array}{c}\text { Progiciel } \\
\text { Tableur } \\
\text { CRM }\end{array}$ & $\begin{array}{c}\text { Progiciel } \\
\text { Tableur } \\
\text { CRM }\end{array}$ \\
\hline Nature du club & GCVE & GCVE & CDN & $\mathrm{CDN}$ & CDN & CDN & PCR & PCR \\
\hline $\begin{array}{l}\text { Dynamique } \\
\text { sur la période }\end{array}$ & $\begin{array}{c}\text { Consolidation } \\
\text { du statut }\end{array}$ & $\begin{array}{c}\text { Consolidation } \\
\text { du statut }\end{array}$ & $\begin{array}{c}\text { Juste derrière } \\
\text { clubs européens }\end{array}$ & $\begin{array}{c}\text { Remontée en } \\
\text { L1 }\end{array}$ & $\begin{array}{c}\text { Succès en L1 } \\
\text { inattendu }\end{array}$ & $\begin{array}{c}\text { Deuxième } \\
\text { partie de L1 }\end{array}$ & $\begin{array}{c}\text { Passage } \\
\text { de L2 à L1 }\end{array}$ & $\begin{array}{l}\text { Deuxième partie } \\
\text { de la L2 }\end{array}$ \\
\hline
\end{tabular}




\section{Les résultats de l'enquête sur le contrôle de gestion des clubs}

Les résultats des entretiens réalisés avec les experts et dans les huit clubs sont présentés, structurés par thèmes et regroupés par dimensions agrégées. Ensuite un recensement des outils de contrôle de gestion mis en œuvre dans les clubs est présenté et une synthèse comparative de l'instrumentation du contrôle de gestion dans les clubs est effectuée.

\subsection{Le pilotage de la performance dans les clubs de football professionnel}

Au cours des entretiens réalisés, que ce soit avec les experts ou dans les clubs, plusieurs thèmes sont apparus particulièrement significatifs.

\subsubsection{L'appareil administratif}

Pour les experts de la gestion administrative et financière du football professionnel, extérieurs aux clubs mais en constant rapport avec eux, le diagnostic fait consensus. Comme le remarque Mme E (salariée à l'UCFP), les moyens humains des clubs sont généralement très limités : «Les clubs sont des PME souvent sous-staffées et avec des gens qui travaillent dans l'urgence et n'ont pas le temps de s'interroger sur leurs modes de décision. Vous avez en général cinq à dix administratifs au total, y compris le commercial, avec un DAF qui gère au quotidien une quantité de problèmes. » (Verbatim 1$)$

Ou encore, d'après Mr G, universitaire :

«La gestion de la plupart des clubs de seconde division est en général très différente de celle des clubs de première division. Elle fait penser à ce qui se passe dans les petites entreprises familiales avec un pilotage à vue. » (V2)

\subsubsection{La perception et la gestion du risque}

La perception et la gestion du risque sont très différentes selon la situation du club : faibles pour les situations stables, fortes si les enjeux sont importants. Pour certains, comme le DG du club C1 :

«Notre ambition sportive est claire : c'est d'être dans les cinq premiers. En France, il y a cinq grands clubs avec des structures importantes et des développements dans tous les domaines: Paris, Lyon, Marseille, Lille et Bordeaux. 
Normalement, nous devons aller dans une compétition européenne le plus souvent possible. »(V3)

Pour les clubs installés en L2, les choses semblent aussi assez claires. L'objectif sportif du club C8 est de rester dans la première partie de L2 tandis que l'objectif économique est l'équilibre :

«Les actionnaires ont tous fait une croix sur leur participation en terme de valeur. Ce sont des entreprises qui ont déprécié cela dans leurs comptes. Ce qu'ils cherchent à éviter, c'est de remettre de l'argent. Ils en ont déjà remis tous il y a trois ou quatre ans (le même montant pour respecter la parité entre eux). » (V4)

Mais pour les clubs qui sont près des seuils : entre compétitions européennes et seulement L1, entre L1 et L2 ou entre L2 et National, la perception du risque est forte. Ainsi, le DG de C6 s'inquiète :

«Il faudrait réduire la volatilité sportive. On monte, on descend: c'est une catastrophe. On a les cas de Metz, Strasbourg ou Grenoble. Aujourd'hui, il est très difficile de descendre en $L 2$ et de remonter immédiatement. Il faut avoir un actionnaire très solide qui puisse donner au club les moyens de passer l'année en L2 en gardant les structures du club. C'est le problème au Mans, au Havre ou à Lens. Si ces clubs font deux ou trois ans en L2, les actionnaires s'épuisent.» (V5)

La DAF du club C4 (anciennement en L1, en L2 lors de l'enquête, et aujourd'hui de nouveau en L1) constate :

«Nous sommes un club qui avec le dimensionnement de ses structures ne peut pas être rentable tant qu'il est en ligue 2 ou alors il faudrait couper au moins la moitié du personnel administratif et réduire le centre de formation. Mais cela voudrait dire, renoncer à nos ambitions.» (V6)

D'autres clubs font le choix de développer leur «agilité » avec une structure adaptable. Dans le club C5, plutôt que de se doter d'un appareil important, le choix a été fait de variabiliser le maximum de charges et d'adopter une structure légère. Une large part des rémunérations des joueurs est variable $(50 \%)$ et donc proportionnelle aux résultats sportifs :

«C'est un principe de gestion et le club refuse un joueur qui ne l'accepte pas. Cela n'a pas été facile au départ, mais les joueurs que nous attirons viennent majoritairement des divisions inférieures. »(V7) 


\subsubsection{Le système d'information comptable au sens large}

Les documents comptables à établir peuvent être nombreux. Le club $\mathrm{C} 2$, doit établir ses informations comptables sous quatre formats : le système de pilotage interne au club, les informations pour la DNCG (assez agrégées et aux normes PCG), les informations pour le fair-play financier demandées par l'UEFA, les informations aux normes consolidées IFRS pour la holding du groupe qui contrôle le club :

«A chaque fois, c'est du retraitement. Souvent, on part de notre document, puis, on établit les éléments pour la DNCG et pour le fair-play (ce n'est pas forcément très cohérent). Après, ce sont les comptes légaux, les comptes consolidés, les documents classiques. »(V8)

Pour le club C3, le plan à 3 ans demandé par la DNCG est quelque chose d'assez formel :

«Le plan à 3 ans, c'est très compliqué. Les prévisionnels à 3 ans sont justes des objectifs d'" enveloppe». Je dirais que ce n'est pas très travaillé. Ce sont des objectifs qui ne sont jamais tenus. »(V9)

Ce plan n'est pas «un exercice simple à réaliser» non plus pour un club comme $\mathrm{C} 4$ qui essaie «de remonter en ligue $1 »$ :

"Il y a vraiment beaucoup de variables qui sont hypothétiques et de ce fait l'exercice n'est pas très réaliste dans notre situation. En général on fait un plan où la première année, on est encore en ligue 2 et à partir de la seconde en ligue 1.» (V10)

Le DG de C6 explique la démarche budgétaire. Il ne part pas d'un objectif de résultat équilibré :

«Au vu de la structure, de la masse salariale et des projets de départs et d'arrivée des joueurs, il faudrait être au moins $6^{\text {ème }}$ pour équilibrer. Si on fait toute la saison sur cette base, on constate fin mai qu'au lieu d'être $6^{\text {ème }}$, on est $12^{\text {ème }}$ et à un mois de la clôture, on ne peut plus rien faire. Je ne fais pas comme cela. J'estime avec le président, mes adjoints et le directeur

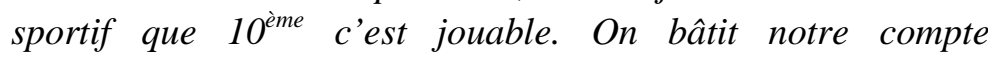
d'exploitation et notre projection de bilan sur cette place là et on dit au président: "Voilà, il manquera tant. Qu'est-ce que l'on fait? On prévoit des ventes de joueurs? Tu remets de l'argent? On augmente le capital?». C'est lui, en tant que président, qui décide. »(V11)

De façon assez proche, ce qui compte pour le club C3, c'est la 
sensibilité des charges au classement :

«Ce qui est important, ce n'est pas nécessairement de se fixer un objectif de classement mais c'est de savoir quelle est la sensibilité de votre résultat au classement. On a une idée de l'impact du classement sur le CA et sur les charges. Nous, en général, on retient un classement de $7^{\text {ème }}$ ou $8^{\text {ème }}$ place, et si on est $4^{\text {ème }}$ ou $5^{\text {ème }}$, on sait quel est l'impact. » (V12)

La formalisation des procédures de gestion proposée par la DNCG est assez peu utilisée dans la pratique d'après une experte membre de l'UCFP :

«On a interrogé les clubs. Le document élaboré pour la DNCG est une base statistique pour la ligue mais en général ne va pas être utilisé par les clubs pour leur gestion quotidienne. »(V13)

\subsubsection{La prévision budgétaire}

Pour les prévisions budgétaires, la démarche du DAF du club C3 est simple :

«Pour établir les prévisions commerciales, cela va être fait avec le directeur commercial. Pour mettre au point les prévisionnels en termes de salaires, cela va être fait avec les différents interlocuteurs concernés. Pour les charges externes, je vais travailler notamment sur les frais de déplacement de l'équipe pro et l'organisation des matchs avec les différents interlocuteurs. Pour le reste, je le fais seul. »(V14)

Au niveau du contrôle de gestion, le responsable financier du club C2 a mis en place des outils simples pour :

«... suivre chaque événement et réviser le budget : que ce soit quand on constate à Noël qu'on va finir $15^{\text {ème }}$ ou que l'on a besoin d'acheter un joueur. On travaille sur Sage en comptabilité et le logiciel Cegid pour la partie analytique et la remise des soldes à la DNCG. La comptabilité analytique est répartie dans chaque budget et on peut analyser les écarts sans avoir à tout retraiter. Mais, on raisonne sur la durée et on ne cherche pas la rentabilité sur le court terme. On n'est pas focalisé sur les coûts. ». Les budgets sont «pessimistes pour la DNCG et en général, on fait $5 \%$ à $10 \%$ de dépenses en moins que le budget annoncé. » (V15)

En matière de comptabilité de gestion et de gestion budgétaire, la situation est différente selon la nature des compétitions et la facilité de prévision (budgétisation assez simple pour les épreuves à points sur la 
durée de type championnat, beaucoup plus difficile dans les épreuves à élimination directe de type coupe qui en général ne sont pas budgétisées) comme l'a démontré Touchais (2001). Des analyses croisées sont généralement réalisées en prenant en compte la diversité des produits et des charges, indique $\mathrm{Mr} \mathrm{H}$, commissaire aux comptes :

«Dans l'analyse des coûts, certains clubs vont faire des analyses par types d'épreuves sportives et d'autres, par centres de responsabilité opérationnelle. Généralement on croise les deux: championnat, coupe de France et coupe de la ligue, voire coupe européenne d'une part ; équipe première, reste de la section professionnelle, frais de structure, etc. d'autre part. $»(\mathrm{~V} 16)$

3.1.5. Le suivi des ressources et des dépenses

L'image de marque et la notoriété de C6 sont relativement faibles et sa zone de chalandise est assez étroite. Le DG indique :

«Les sources de revenus sont dans l'ordre: les droits TV (il faut satisfaire la ligue et derrière Canal + et BeIn Sport); les sponsors qui doivent avoir un produit inattaquable et de bonne facture; les spectateurs. Il y a deux types de spectateurs: les fidèles (supporters, ultras, clubs amateurs) qui sont gérés par le président pour qu'ils viennent et participent à la bonne ambiance des matchs et les autres qui consomment ce spectacle plutôt qu'un autre et que les commerciaux et moi, on doit faire venir. » (V17)

Le responsable financier de $\mathrm{C} 7$ remarque :

«Pour la prévision et le suivi budgétaire match par match, c'est compliqué. Dans la masse salariale, on a une partie variable liée à la prime de match, assez importante (de 25000 $€$ à $50000 €$ dans certains cas). Pour les recettes, on fait des estimations match par match de la fréquentation et des produits. Pour un match avec Marseille, on a un coefficient 1,81 par rapport à la moyenne. Pour d'autres matchs, c'est 0,27. On a le budget, le réel et j'analyse les écarts. Les déplacements des pros à l'extérieur, c'est vu match par match. Même chose pour la sécurité à domicile. Pour le suivi, je communique avec le président à certains moments précis. En interne, l'autonomie des responsables est très limitée et il a peu de budgets délégués sauf pour le responsable de la boutique. C'est moi qui budgétise tout après une discussion avec chacun 
des responsables. » (V18)

Certains processus sont particulièrement cruciaux pour les clubs : le processus de recrutement d'un joueur (aspects juridiques, fiscaux, administratifs), le processus médical (examen médical avec gestion du secret médical, suivi du mode de vie, problèmes de dopage, etc.), le processus d'organisation des matchs (billetterie et fraude à l'entrée, sécurité, gestion des divers sponsorings, etc.). Plusieurs indicateurs cruciaux sont généralement suivis en parallèle avec des rythmes de reporting différents comme l'indique $\mathrm{Mr} \mathrm{H}$, Commissaire aux comptes :

«Le tableau de bord commercial est hebdomadaire. Le tableau de bord de trésorerie suit les encaissements au jour le jour. Le tableau de bord de la masse salariale est quotidien entre le 15 mai et fin juillet: c'est à ce moment que les joueurs entrent et sortent des effectifs du club. Quand on signe un contrat, on met à jour. Pour un club de L1, le président peut suivre une quinzaine de chapitres budgétaires et cela suffit. »

Et certains éléments sont toujours pris en compte :

«Dans tous les clubs, il y a un suivi des recettes spectateurs avec un état quantitatif après chaque match, tribune par tribune. »(V19)

\subsubsection{L'organisation du reporting}

$\mathrm{Au}$ club $\mathrm{C} 4$, des prévisions budgétaires assez fines sont faites et des arbitrages réalisés en cours de saison (par exemple déplacement pour un match en avion ou en car). Par prudence, les matchs de coupe ne sont pas budgétés (ni pour les recettes, ni pour les dépenses) sauf le premier tour. La DAF indique :

«J'ai mis en place un suivi détaillé des charges en parallèle avec le budget. Comme cela le DG aura un état de la situation avant de contresigner une commande. Ce n'est pas toujours facile à réaliser car ce sont les responsables de chaque service qui font les commandes et qui vont devoir remplir le tableau. Ils ont un sentiment de flicage. Le contrôleur de gestion doit être pédagogue, expliquer et réexpliquer. » (V20)

Un reporting mensuel global et détaillé par centre de coût est remis chaque mois au président avec l'explication des variations. Le système de gestion semble cohérent et adapté à la ligue 1 qui est visée :

«On est arrivé à un niveau où on a tous les indicateurs nécessaires. Lorsque le président me demande telle ou telle 
information, je peux la lui envoyer tout de suite selon la présentation qu'il souhaite. Le contrôleur de gestion et moi, nous sommes très réactifs. Lui, il est issu du foot et moi, je suis plus technicienne. On a un bon équilibre.» (V21)

La DAF du club C4 supervise trois comptables et un contrôleur de gestion. Tout est fait en interne sauf la gestion des stadiers qui est effectuée par une agence d'intérim. La trésorerie est très suivie :

«Toutes les semaines, un tableau de suivi trésorerie sur un mois roulant est envoyé au président. Aujourd'hui le club ne vit que grâce à lui. Chaque mois, il fait des apports en compte courant en fonction des besoins. » (V22)

\subsubsection{L'outillage du contrôle de gestion}

Comme la flexibilité financière du club C5 est très grande, il y a peu d'instrumentation de gestion :

«On est dans une formule atypique. On n'a pas les outils de gestion traditionnels avec coûts complets, etc. On a notre propre outil de prévision et de simulation qui permet de prévoir en fonction du nombre de points au classement le niveau de nos dépenses et de nos recettes en temps réel. » (V23)

Les opérationnels du club ont des tableaux de bord privatifs et les remontées d'informations à la direction générale sont systématiques mais peu formalisée :

«Plus que des tableaux de bord, ce que l'on a c'est un partage permanent de l'information en s'appuyant sur le réseau informatique. Il y a des check-list des opérations à réaliser à $J$ 8, à $J-4$, etc. » (V24)

Le DAF du club C3 indique :

«On peut toujours faire mieux en matière de formalisation de la gestion. La seule question est : Pour faire quoi? Et combien cela coûterait? Pour avoir un suivi plus fin, cela va nécessiter un outil de gestion informatique plus costaud, un poste de plus et du temps de DAF pour un rendement que je juge intuitivement assez faible. Je préfère donc en rester à une approche plus rustique et maîtriser la globalité des charges plutôt que de faire des analyses sans fin et établir des ratios qui vont un peu noyer tout le monde. »(V25)

Nous pouvons synthétiser toutes ces informations dans le schéma 2. 
Schéma 2: Structuration des verbatims recueillis

\begin{tabular}{|c|}
\hline $\begin{array}{l}\text { CONCEPTS DE 1er ORDRE : } \\
\text { VERBATIMS DE TERRAIN }\end{array}$ \\
\hline $\mathrm{V} 1$ : clubs sous-staffés \\
\hline V2: gestion de petits clubs comme des PME \\
\hline $\begin{array}{l}\text { V3: possibilité de développer une structure } \\
\text { stable pour les grands clubs }\end{array}$ \\
\hline $\begin{array}{l}\text { V4: pour les petits clubs pas d'ambition } \\
\text { financière en général }\end{array}$ \\
\hline v5 : la volatilité sportive est catastrophique \\
\hline $\begin{array}{l}\mathrm{V} 6 \text { : problème de dimensionnement quand } \\
\text { on oscille entre L1 et L2 }\end{array}$ \\
\hline $\begin{array}{l}\text { V7: choix possible de variabiliser } \\
\text { les charges }\end{array}$ \\
\hline $\begin{array}{l}\text { V8: } 4 \text { formats comptables à établir pour un } \\
\text { grand club }\end{array}$ \\
\hline V9: le plan à 3 ans est juste indicatif \\
\hline V10 : le plan à 3 ans est très hypothétique \\
\hline V11: le budget dépend des moyens \\
\hline $\begin{array}{l}\text { V12 : il faut anticiper limpact budgétaire du } \\
\text { classement sportif }\end{array}$ \\
\hline $\begin{array}{l}\text { V13: le document pour la DNCG ne sert pas } \\
\text { pour la gestion }\end{array}$ \\
\hline $\begin{array}{l}\text { V14: prévisions bugdétaires établies assez } \\
\text { simplement }\end{array}$ \\
\hline $\begin{array}{l}\text { V15: pas de focalisation sur les coûts } \\
\text { d'exploitation à court terme }\end{array}$ \\
\hline V16: budgétisation différenciée \\
\hline $\begin{array}{l}\text { V17 : supporters gérés par le président, } \\
\text { spectateurs par le marketing } \\
\text { V18: suivi des produits et des charges à } \\
\text { chaque match } \\
\text { V19: suivi détaillé de tous les processus } \\
\text { opérationnels et du recrutement }\end{array}$ \\
\hline V20: système d'autorisation des dépenses \\
\hline $\begin{array}{l}\text { V21 : grand nombre d'indicateurs } \\
\text { disponibles }\end{array}$ \\
\hline $\begin{array}{l}\text { V22: tableaux de bord hebdomadaires de } \\
\text { trésorerie }\end{array}$ \\
\hline $\begin{array}{l}\text { V23 : club axé sur la flexibilité : pas de coûts } \\
\text { complets }\end{array}$ \\
\hline $\begin{array}{l}\text { V24: base de données mais pas de tableaux } \\
\text { de bord formalisés }\end{array}$ \\
\hline $\begin{array}{l}\text { V25 : refus d'outils de gestion trop } \\
\text { sophistiqués }\end{array}$ \\
\hline
\end{tabular}

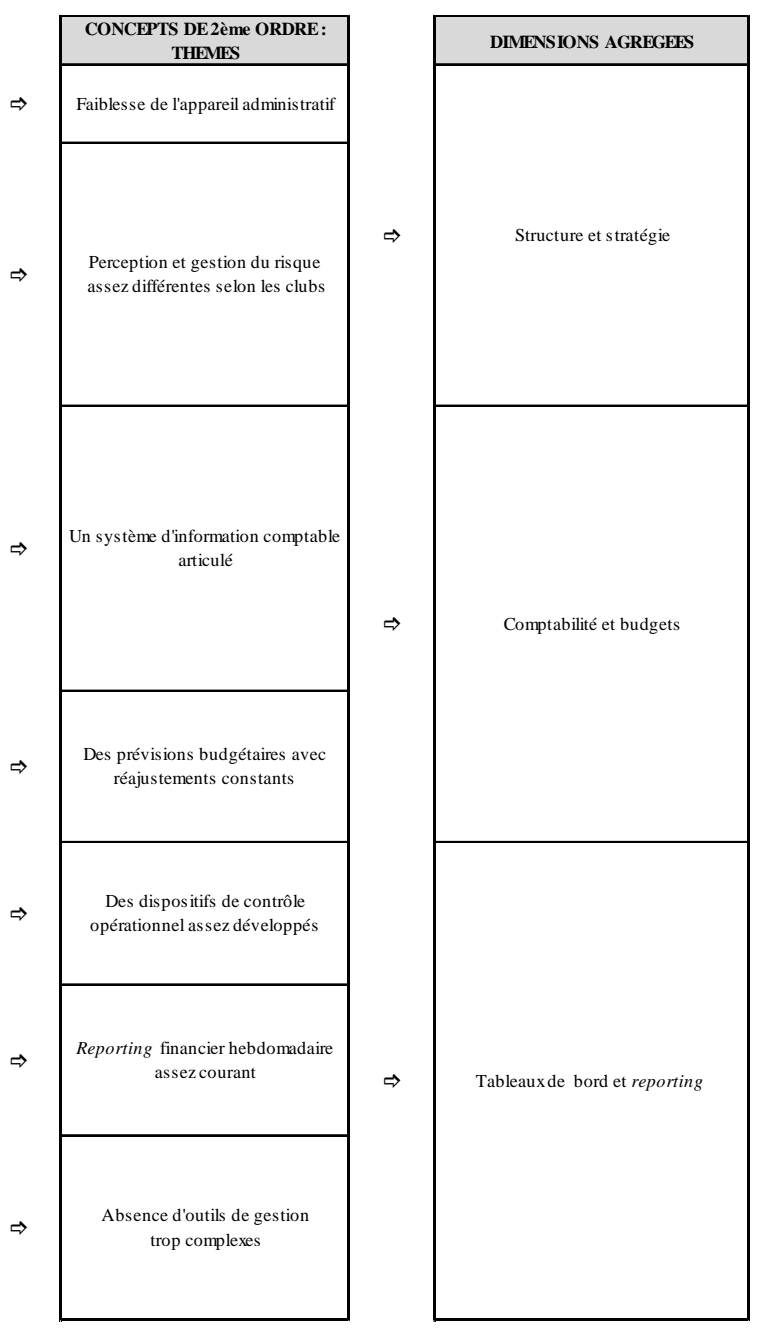

\subsection{L'instrumentation du contrôle de gestion dans les clubs}

Les outils du contrôle de gestion des clubs de football professionnels français qui nous sont apparus comme assez couramment utilisés dans les huit clubs étudiés peuvent être regroupés sous quatre rubriques principales et leur fréquence d'utilisation et la nature de leur usage par la direction peuvent être mises en évidence. 
Tableau 3: Les outils de contrôle de gestion utilisés dans les clubs

\begin{tabular}{|c|c|c|c|}
\hline AXES DE GESTION & $\begin{array}{c}\text { INSTRUMENTS } \\
\text { DEPLOYES }\end{array}$ & $\begin{array}{c}\text { FREQUENCE } \\
\text { D'UTILISATION }\end{array}$ & OBJECTIF \\
\hline \multicolumn{4}{|l|}{ Finance } \\
\hline $\mathrm{F} 1$ & Tableaux de la DNCG & Mai et septembre & Légitimité ext. \\
\hline $\mathrm{F} 2$ & Fair play financier & Annuelle (saison) & Légitimité ext. \\
\hline F3 & Comptes de groupe & Annuelle (année) & Légitimité int. \\
\hline $\mathrm{F} 4$ & Reporting comptable / groupe & Mensuelle & Légitimité int. \\
\hline F5 & Suivi et actualisation budgets & En continu & Animation int. \\
\hline F6 & Suivi de la trésorerie & Quotidienne & Aide / décision \\
\hline F7 & Suivi des investissements & Selon réalisation & Aide / décision \\
\hline F8 & Compta. analytique & Mensuelle & Aide / décision \\
\hline F9 & Suivi des filiales & Mensuelle & Animation int. \\
\hline \multicolumn{4}{|l|}{ Marketing } \\
\hline M1 & Suivi contrats et partenariats & Mensuelle & Aide / décision \\
\hline M2 & Abonnements en pré-saison & Hebdomadaire & Aide / décision \\
\hline M3 & Suivi des recettes au stade & Après les matchs & Aide / décision \\
\hline M4 & Suivi recettes diversification & Mensuelle & Aide / décision \\
\hline M5 & Suivi revenus merchandising & Mensuelle & Aide / décision \\
\hline \multicolumn{4}{|l|}{ Production } \\
\hline $\mathrm{P} 1$ & Suivi des charges & Hebdomadaire & Aide / décision \\
\hline $\mathrm{P} 2$ & Analyse coûts d'un match & Annuelle (saison) & Aide / décision \\
\hline P3 & Analyse par compétition & Annuelle & Aide / décision \\
\hline P4 & Suivi sécurité et logistique & Annuelle & Aide / décision \\
\hline P5 & Suivi abonnés et supporters & Périodique & Légitimité ext. \\
\hline \multicolumn{4}{|l|}{$\mathbf{R H}$} \\
\hline H1 & Suivi financier recrutement & Lors des mercatos & Aide / décision \\
\hline $\mathrm{H} 2$ & Suivi médical des joueurs & Permanent & Aide / décision \\
\hline H3 & Veille externe / recrutements & Quotidienne & Aide / décision \\
\hline $\mathrm{H} 4$ & Suivi du centre de formation & Mensuelle & Animation int. \\
\hline
\end{tabular}

La fréquence d'utilisation est essentiellement annuelle et vise à la légitimité interne et externe pour les instruments comptables et est mensuelle avec un objectif d'animation interne pour les budgets. Les outils du contrôle de gestion commercial ont une fréquence mensuelle et surtout hebdomadaire et servent à l'aide à la décision. Dans le domaine des processus productifs, la plupart des outils visent à l'aide à la décision avec des fréquences assez variables très liées au rythme des compétitions. Le capital humain des clubs est géré avec beaucoup de soins que ce soit lors des mercatos d'été et d'hiver (choix d'investissement dans les joueurs) ou pendant la saison (gestion des effectifs par l'entraineur). L'usage qui est fait de ces instruments du contrôle de gestion dans les huit clubs de notre étude va maintenant être présenté dans le tableau 4 ci dessous en reprenant les outils du tableau précédent. 
Tableau 4: Degré d'instrumentation du contrôle de gestion des huit clubs étudiés

\begin{tabular}{|l|l|l|l|l|l|l|l|l|l|}
\hline \multicolumn{1}{|c|}{ AXES } & C1 & C2 & C3 & C4 & C5 & C6 & C7 & C8 & Taux d'usage \\
\hline F1 DNCG & $\mathrm{X}$ & $\mathrm{X}$ & $\mathrm{X}$ & $\mathrm{X}$ & $\mathrm{X}$ & $\mathrm{X}$ & $\mathrm{X}$ & $\mathrm{X}$ & $\mathbf{8 / 8}$ (obligatoire) \\
F2 Fair play & $\mathrm{X}$ & $\mathrm{X}$ & & & & & & & $\mathbf{2 / 8}$ (contingent) \\
F3 Comptes groupe & $\mathrm{X}$ & $\mathrm{X}$ & $\mathrm{X}$ & & & & & & $\mathbf{3 / 8}$ (contingent) \\
F4 Reporting groupe & $\mathrm{X}$ & $\mathrm{X}$ & $\mathrm{X}$ & & & & & & $\mathbf{3 / 8}$ (contingent) \\
F5 Suivi et actual.budg. & $\mathrm{X}$ & $\mathrm{X}$ & $\mathrm{X}$ & $\mathrm{X}$ & $\mathrm{X}$ & & & $\mathrm{X}$ & $\mathbf{6 / 8}$ \\
F6 Suivi de la trésorerie & $\mathrm{X}$ & $\mathrm{X}$ & $\mathrm{X}$ & $\mathrm{X}$ & $\mathrm{X}$ & $\mathrm{X}$ & $\mathrm{X}$ & $\mathrm{X}$ & $\mathbf{8 / 8}$ \\
F7 Suivi des investiss. & $\mathrm{X}$ & $\mathrm{X}$ & $\mathrm{X}$ & & $\mathrm{X}$ & $\mathrm{X}$ & $\mathrm{X}$ & & $\mathbf{6 / 8}$ \\
F8 Compt. ana. multiaxes & $\mathrm{X}$ & $\mathrm{X}$ & & $\mathrm{X}$ & & & & & $\mathbf{3 / 8}$ \\
F9 Suivi des filiales & $\mathrm{X}$ & $\mathrm{X}$ & & $\mathrm{X}$ & $\mathrm{X}$ & & & & $\mathbf{4 / 8}$ \\
\hline C1 Contrats et parten. & $\mathrm{X}$ & $\mathrm{X}$ & $\mathrm{X}$ & $\mathrm{X}$ & $\mathrm{X}$ & $\mathrm{X}$ & $\mathrm{X}$ & & $\mathbf{7 / 8}$ \\
C2 Abonnements & $\mathrm{X}$ & $\mathrm{X}$ & $\mathrm{X}$ & $\mathrm{X}$ & $\mathrm{X}$ & $\mathrm{X}$ & $\mathrm{X}$ & $\mathrm{X}$ & $\mathbf{8 / 8}$ (nécessaire) \\
C3 Recettes matches & $\mathrm{X}$ & $\mathrm{X}$ & $\mathrm{X}$ & $\mathrm{X}$ & $\mathrm{X}$ & $\mathrm{X}$ & $\mathrm{X}$ & $\mathrm{X}$ & $\mathbf{8 / 8}$ (nécessaire) \\
C4 Recettes activ.divers. & $\mathrm{X}$ & $\mathrm{X}$ & & $\mathrm{X}$ & & & & & $\mathbf{3 / 8}$ \\
C5 Recettes merchand. & $\mathrm{X}$ & $\mathrm{X}$ & $\mathrm{X}$ & $\mathrm{X}$ & $\mathrm{X}$ & $\mathrm{X}$ & & & $\mathbf{6 / 8}$ \\
\hline P1 Suivi des charges & $\mathrm{X}$ & $\mathrm{X}$ & $\mathrm{X}$ & $\mathrm{X}$ & $\mathrm{X}$ & $\mathrm{X}$ & $\mathrm{X}$ & $\mathrm{X}$ & $\mathbf{8 / 8}$ \\
P2 Coûts d'un match & $\mathrm{X}$ & $\mathrm{X}$ & $\mathrm{X}$ & $\mathrm{X}$ & $\mathrm{X}$ & $\mathrm{X}$ & $\mathrm{X}$ & $\mathrm{X}$ & $\mathbf{8 / 8}$ \\
P3 Coûts par compét. & $\mathrm{X}$ & & & & & & & & $\mathbf{1 / 8}$ \\
P4 Sécurité et logistique & $\mathrm{X}$ & $\mathrm{X}$ & $\mathrm{X}$ & $\mathrm{X}$ & $\mathrm{X}$ & $\mathrm{X}$ & $\mathrm{X}$ & $\mathrm{X}$ & $\mathbf{8 / 8}$ \\
P5 Abonnés-supporters & $\mathrm{X}$ & $\mathrm{X}$ & $\mathrm{X}$ & $\mathrm{X}$ & $\mathrm{X}$ & $\mathrm{X}$ & & & $\mathbf{6 / 8}$ \\
\hline H1 Impact fi. recrut. & $\mathrm{X}$ & $\mathrm{X}$ & $\mathrm{X}$ & $\mathrm{X}$ & $\mathrm{X}$ & $\mathrm{X}$ & $\mathrm{X}$ & $\mathrm{X}$ & $\mathbf{8 / 8}$ (nécessaire) \\
H2 Suivi médical et sport. & $\mathrm{X}$ & $\mathrm{X}$ & $\mathrm{X}$ & $\mathrm{X}$ & $\mathrm{X}$ & $\mathrm{X}$ & $\mathrm{X}$ & $\mathrm{X}$ & $\mathbf{8 / 8}$ (nécessaire) \\
H3 Veille ext. pour recrut. & $\mathrm{X}$ & $\mathrm{X}$ & $\mathrm{X}$ & $\mathrm{X}$ & $\mathrm{X}$ & $\mathrm{X}$ & & & $\mathbf{6 / 8}$ \\
H4 Centre de formation & $\mathrm{X}$ & $\mathrm{X}$ & $\mathrm{X}$ & $\mathrm{X}$ & $\mathrm{X}$ & $\mathrm{X}$ & $\mathrm{X}$ & $\mathrm{X}$ & $\mathbf{8 / 8}$ (obligatoire) \\
\hline Total / 23 club & $\mathbf{2 3}$ & $\mathbf{2 2}$ & $\mathbf{1 8}$ & $\mathbf{1 8}$ & $\mathbf{1 7}$ & $\mathbf{1 5}$ & $\mathbf{1 2}$ & $\mathbf{1 1}$ & \\
\hline
\end{tabular}

On voit bien que certains outils sont très répandus soit qu'ils soient obligatoires (documents pour la DNCG), soit qu'ils soient jugés nécessaires par tous (suivi des abonnements, de la recette au stade les jours de match, la fiche de santé des recrutés ou le suivi physique des joueurs de l'équipe première). Certains autres sont contingents par nature: le fair play UEFA pour les participants aux coupes européennes ou les comptes détaillés pour les filiales de groupes. Pour le reste les choix mettent en évidence le lien avec la typologie proposée des clubs dans la partie 1 : les grands clubs sont dotés de la panoplie quasi-complète des outils (22 ou 23/23); les clubs moyens ont un nombre importants d'outils (de 15 à 18/23); les petits clubs sont moins instrumentés surtout dans la dimension financière mais utilisent assez souvent les mêmes outils dans l'exploitation (11 ou 12/23). 


\section{Les enseignements de l'étude du contrôle de gestion des clubs}

Nous allons analyser maintenant de façon synthétique ce qui a trait au mode de fonctionnement des clubs et à leur outillage de contrôle de gestion.

\subsection{La plupart des clubs de football professionnel n'ont en matière financière pour objectif que l'équilibre}

Dans la majorité des clubs rencontrés, la maximisation de leur valeur financière n'est pas un objectif envisagé car cela leur semble peu réalisable à court terme. Selon un DG s'exprimant sur les attentes du propriétaire :

«Mon objectif est juste d'équilibrer les comptes. Mon président ne me demande pas de gagner de l'argent. Il ne serait pas contre mais ce n'est pas sa priorité. Aujourd'hui il serait content que j'arrive à ce que le club ne lui coûte plus d'argent. C'est un président supporter.» (V26)

Les clubs de football sont donc des institutions à finalités plus multiples et à gouvernance plus complexe que les entreprises qui cherchent à maximiser leur valeur financière. Par ailleurs, comme leur activité sportive est par nature organisée et co-produite avec les autres clubs, il leur est très difficile de développer des stratégies originales de création de valeur économique. Les clubs ont donc des caractéristiques spécifiques qui les distinguent des autres entreprises de spectacle et limitent leur expansion économique (sauf cas très particuliers du type PSG actuellement et demain peut-être Monaco). Cela a des conséquences sur le système de pilotage de la performance économique qui reste en général subordonné au système de pilotage de la performance sportive. Touchais (2000, p. 19) concluait un article sur le contrôle de gestion dans les clubs de football professionnel par le pronostic suivant :

«A terme la diversification doit dégager plus de bénéfices permettant le financement du sport spectacle et la distribution de dividendes. Lorsque cette évolution aura abouti, le contrôle de gestion aura changé de visage. Ses particularités actuelles vont probablement s'atténuer pour se rapprocher d'entreprises plus classiques ».

Quatorze ans après, ce n'est pas ce que l'on constate ... 


\subsection{La régulation de la DNCG encadre la gestion budgétaire des clubs et « éduque » les dirigeants}

La dynamique du contrôle de gestion dans les clubs professionnels a été étudiée par Hasrouri (2006). Il rappelle que les clubs fonctionnent plus comme des institutions que comme des organisations. Cela a des conséquences sur leurs pratiques de gestion. L'encadrement par la LFP et la DNCG, contribue à homogénéiser les pratiques et à «éduquer» les dirigeants et propriétaires de clubs. Un certain isomorphisme organisationnel, coercitif au sens de Di Maggio et Powell (1983), s'exerce sur la gestion des clubs au travers des règles édictées par la DNCG qui permettent de limiter les dérives financières en France. Ce phénomène est conforté par l'UEFA qui impose sa procédure du fair play financier aux clubs participant aux coupes européennes. Cet isomorphisme est accentué par des raisons technologiques à cause de l'utilisation du même logiciel intégré de gestion dans la majorité des clubs. En matière de contrôle de gestion, nous rejoignons en partie les conclusions de Lauzanas (2003, p. 910) :

"Quels que soient les clubs, la DNCG a une influence fondamentale sur la comptabilité générale, les états budgétaires ou les procédures de suivi financier des ressources humaines. L'instrumentation s'inscrit bien dans l'acte de rendre compte et dans un mimétisme coercitif. »

Ceci est vrai pour la plupart des clubs, toutefois les GCVE ont d'autres contraintes vis à vis des holdings et de l'UEFA et il faut rappeler aussi, pour nuancer les choses, que la DNCG n'a pas pour mission de s'immiscer dans la gestion courante des clubs.

\subsection{La cohérence des choix sur le long terme et la gestion des phases de «mercato » sont très importantes}

Si l'optimisation des choix de gestion en cours de saison est nécessaire, la cohérence des choix stratégiques et l'expérience des dirigeants sur la durée sont essentielles (Lechner et Gudmundsson 2012). Comme l'indique un DG rencontré :

«Ce qui permet d'équilibrer les comptes, le signe de bonne gestion, c'est la pérennité. Il faut avoir une gestion sportive qui a un sens, cohérente dans la durée. A partir du moment où vous avez un départ de coach imprévu en fin de saison, vous mettez en péril le club. Nous, quand on a perdu beaucoup d'argent, c'était lié au départ du coach. »(V27) 
Un autre DG explique le fonctionnement de la plupart des clubs :

"Gérer un club c'est facile. Ce qui est difficile c'est de le dimensionner. Le problème, ce sont les périodes de mercato où on est en danger. On peut se planter, acheter un joueur blessé ... on a un joueur ici depuis 18 mois qui n'a jamais joué. C'est une erreur de gestion sportive qui coûte 1,5 millions! »(V28)

Les divers outils d'optimisation des choix et des décisions de gestion (compétences juridiques, techniques de gestion de projets, de choix d'investissement ou de gestion des risques) sont donc utiles notamment lors des périodes autorisées de recrutement de joueurs (mercato). Comme souligné auparavant, le contrôle opérationnel des processus spécifiquement liés au métier dans les phases d'acquisition et de gestion des actifs matériels (immobilier, centre de formation, stade) ou immatériels (joueurs, entraineur) est un élément clé du contrôle de gestion des clubs professionnels. La prise en compte des aspects contextuels permettant une gestion des clubs au plus près du terrain est alors indispensable par delà les outils de modélisation financière classiques du contrôle de gestion (comptabilité de gestion et budgets).

\subsection{La variabilisation des charges permet à certains clubs d'être adaptables et flexibles}

Un des moyens possibles pour diminuer le risque financier généré par un échec (ou parfois par un succès !) sportif, c'est de concevoir le club comme un nœud de contrats. L'entreprise sportive va recourir à des «mécanismes de type marché ». Elle ne va pas forcément chercher à développer son appareil administratif mais plutôt à alléger et variabiliser le maximum d'éléments du compte de résultat (produits et charges) mais aussi du bilan (actifs et financement liés). Néanmoins, l'externalisation a aussi ses limites. Un DAF témoigne sur le mode de fonctionnement des petits clubs :

«Dans les petits clubs de L2, le DAF fait tout sauf de la finance. La comptabilité est sous-traitée à un cabinet. Il fait surtout de l'administratif, le lien avec les sous-traitants, etc. En L1, on trouve cela dans quelques clubs aussi mais, en général les clubs sont plus structurés. »(V29)

Par ailleurs, une forte variabilité des salaires semble ne pas être suffisante pour dégager un bénéfice :

«Il est très difficile de faire coöncider rentabilité et performance sportive. Lorient est un bon compromis : jouer le milieu du classement et gagner de l'argent. Mais il est difficile 
quand on est ambitieux de gagner de l'argent. »(V30)

Lauzanas (2003, p. 916) s'interrogeait sur l'instrumentation des clubs d'une certaine taille :

«Les dirigeants des clubs semblent moins percevoir l'incertitude tout en disposant de plus d'outils. Quel sens donner à cette relation? L'existence d'outils efface-t-elle la perception de l'incertitude ou bien faut-il remettre en cause l'idée selon laquelle le moteur de l'apparition de l'outil est l'incertitude contre laquelle il faut lutter? »

En réalité, ne faut-il pas renverser l'analyse et comprendre que quand l'incertitude est forte les clubs s'engagent le moins possible et donc évitent les risques non pas en montant un «appareil managérial» mais en plafonnant l'envergure du club? Ils recourent aux contrats, aux partenariats, aux sous-traitants, aux formules de primes acceptées transitoirement par les joueurs (la main invisible des marchés). La gestion interne et les outils de pilotage de la performance économique ne seront pas trop développés et la chaîne de valeur sera réduite en externalisant tout ce qui est possible et en flexibilisant au maximum les paramètres de gestion. Par contre, quand ils ont plus de confiance, ils investissent sur la durée (appareil administratif, centre de formation, stade, contrats avec les joueurs) et donc ils accroissent leur « épaisseur» et la densité des processus opérationnels qu'ils pilotent directement. Il leur faut alors développer la fonction et les outils du contrôle de gestion (la main visible des managers).

\subsection{Seuls les grands clubs structurent un appareil managérial et se dotent d'un système de pilotage global}

Comme dans les PME, les clubs utilisent des indicateurs physiques et financiers dans des tableaux de bord de terrain mis en œuvre de façon plus ou moins autonome et privative par les managers opérationnels dans les fonctions logistique, commerciale ou RH. Certains indicateurs sont transmis au DG et à l'équipe de direction, soit de façon régulière et fréquente (au lendemain de chaque match ou chaque mois), soit ponctuellement, à la demande. Il existe des différences significatives entre d'une part, les GCVE très structurés $(\mathrm{C} 1$ et $\mathrm{C} 2)$ et les $\mathrm{CDN}$ dotés d'un appareil de gestion moins large (C3, C4, C5, C6), et d'autre part, les PCR (C7 et C8). Ces derniers sont dans des aires urbaines plus petites. Ils ont un CA moyen plus réduit et des effectifs plus restreints. Il y a un écart net de situation entre ces deux catégories de clubs. Ceci est manifeste aussi dans l'instrumentation du contrôle de gestion, 
beaucoup plus réduite dans les petits clubs. Les grands clubs sont dotés d'un système de pilotage de la performance complet (budgets, tableaux de bord stratégique, outils opérationnels et nombreux logiciels, etc.). Les clubs $\mathrm{C} 1, \mathrm{C} 2$ et $\mathrm{C} 3$ utilisent les normes IFRS pour rendre compte à la holding. $\mathrm{C} 1$ fonctionne comme une filiale d'un grand groupe en appliquant les méthodes des grands groupes. Ce sont encore des entreprises moyennes par la taille mais équivalentes aux grandes entreprises par le déploiement des informations comptables et la mise en œuvre des outils assurant la convergence des comportements.

\subsection{La comptabilité de gestion n'est vraiment développée que dans les grands clubs aux activités diversifiées}

La comptabilité de gestion commence à se développer dans les clubs souvent grâce à l'installation d'un logiciel intégré. Le découpage analytique reprend généralement la structure organisationnelle du club et le découpage en centres de responsabilité. Les calculs de coûts existent mais sont peu fréquents et généralement réalisés à partir des chiffres annuels. Seuls les clubs ayant fait le choix de l'intégration du maximum de processus en interne dans leur périmètre et de développement d'activités diversifiées autour de leur cœur de métier (le football professionnel) effectuent des calculs de coûts spécifiques qui sont fréquents, réguliers et objets de reporting. Et, comme le remarque Lauzanas (2003, p. 917): "Une forte proximité avec le monde industriel paraît favorable à l'instrumentation. ». Ceci est surtout vrai pour les outils d'aide à la prise de décision et d'optimisation des choix de gestion (comme les calculs de coûts) et un peu moins net pour les outils permettant d'assurer la convergence des comportements et le pilotage de la performance (comme les budgets avec reporting mensuel, analyse d'écarts, action correctives ou re-prévisions).

\section{Conclusion}

Une vision moderne du contrôle de gestion nécessite de dépasser la seule prise en compte des outils universels de modélisation financière comme la comptabilité de gestion et les budgets pour développer une approche plus large prenant en compte tous les dispositifs formalisés assurant la mise en œuvre du business model jusqu'au niveau des processus opérationnels de terrain. Cette approche plus vaste mais contextualisée par nature, nécessite une démarche contingente prenant en compte les spécificités du métier. Pour mettre en œuvre une telle 
analyse au niveau du secteur du sport spectacle, nous avons analysé théoriquement le métier et la gouvernance des clubs de football professionnel, effectué des entretiens exploratoires auprès d'un panel d'experts en contrôle de gestion dans le football professionnel et réalisé des entretiens et une collecte de l'information documentaire dans huit clubs représentatifs des quarante des ligues 1 et 2 afin de mener une analyse comparée et synthétique.

Les principaux enseignements sont les suivants :

- la plupart des clubs visent l'équilibre financier plus que la performance économique maximale ;

- les structures collectives, via la DNCG, jouent un rôle de régulation des pratiques de gestion budgétaire et incitent donc à la mise place d'un minimum de contrôle de gestion dans les clubs ;

- les processus opérationnels cruciaux qui nécessitent un contrôle strict, se déroulent pendant la saison (matchs) mais aussi hors exploitation pendant le mercato et l'inter-saisons (recrutements, investissements, etc.) ;

- plutôt que de développer leur appareil de contrôle, certains clubs préfèrent variabiliser leurs charges et externaliser le maximum d'activités pour rester flexibles ;

- l'usage d'indicateurs et de tableaux de bord opérationnels est généralisé mais le recours aux tableaux de bord stratégiques et aux systèmes globaux de pilotage de la performance économique est réservé aux grands clubs ;

- la mise en œuvre d'une réelle comptabilité de gestion avec usage régulier n'est le fait que de certains grands clubs qui structurent leur organisation, internalisent les processus opérationnels et diversifient leurs activités annexes.

Le football professionnel en France est un métier où la finalisation est complexe, où la gouvernance est plurielle et le risque intrinsèque à l'activité. Dans un article consacré au contrôle de gestion dans les clubs de football professionnel publié dans FCS en 2001, Lionel Touchais (2001) faisait le point sur les modes de pilotage de la performance en environnement incertain et il évoquait certaines possibilités d'aménagement des outils budgétaires pour mieux piloter dans ce contexte. Notre étude montre qu'on a intérêt à dépasser la focalisation sur le seul outil budgétaire pour élargir l'analyse à l'ensemble du système de pilotage de la performance financière. Les clubs sont très divers. Les pratiques de contrôle de gestion ont des éléments 
communs : la liasse budgétaire exigée par la DNCG et les outils liés aux fonctions opérationnelles et aux processus spécifiques du métier. Elles se différencient en matière de pilotage de la performance (tableaux de bords autonomes et locaux ou système stratégique global) généralement, en fonction de la taille du club et de son rattachement ou non à une structure supérieure (maison mère ou holding). Par ailleurs, pour maîtriser leurs coûts et rester «agiles », certains clubs (souvent en phase transitoire dans une trajectoire dynamique) s'appuient sur des «mécanismes de type marché »en externalisant le maximum de processus productifs et variabilisant les charges internes restantes alors qu'à l'inverse, de grands clubs bien installés se dotent de leur propre appareil managérial et mettent en œuvre une réelle comptabilité de gestion pour gérer en interne leur chaîne de valeur s'ils sont dans une logique d'acquisition d'actifs, de gestion intégrée des processus et de diversification dans des activités extra-sportives.

Il s'agit là plus d'apports dans le champ de la compréhension empirique que d'une contribution théorique nouvelle mais cela nous semble essentiel. Il nous faudrait maintenant étudier ce qui se passe dans les clubs sportifs professionnels des ligues fermées d'Amérique du Nord. Le business model est assez différent, la diversification très développée autour de marques fortes et l'instrumentation de gestion d'une ampleur considérable. Notre analyse du contrôle de gestion des clubs sportifs professionnels français gagnerait aussi à être complétée par une étude symétrique dans le champ de l'industrie du divertissement. En effet, certains aspects sont communs (attentes des spectateurs, caractéristiques économiques des prestations, enracinement local, etc.) et d'autres très différents (production autonome dans le cas des représentations culturelles contre co-production entre clubs pour les matchs, liberté de développement dans la culture contre régulation collective dans le sport, etc.). 


\section{Bibliographie}

Andreff W. (2010), Economie internationale $d u$ sport, Presses Universitaires de Grenoble.

Anthony R. (1965), Planning and Control Systems: A Framework for Analysis, Harvard Business Press.

Anthony R. et Govindarajan (1995), Management Control Systems, Irwin, 8 ème édition.

Augé B. (1998), La formalisation de la gestion des clubs sportifs : un essai d'observation et d'interprétation, thèse de Doctorat en Sciences de Gestion, Université de Montpellier.

Bourg J.-F. et Gouguet J.-J. (201), Economie du sport, Editions La Découverte, collection Repères.

Brocard J.-F. (2009), «La problématique des agents sportifs sur le marché du travail des sportifs professionnels » in Fontanel J., Bensahel L. et Chaix P., Regards sur l'économie et le management du sport et des sportifs professionnels, l'Harmattan.

Courtney H., Kirkland J., Viguerie P. (1997), « Strategy under Uncertainty», Harvard Business Review, novembre-décembre, p. 67-79.

Couvelaere V. et Richelieu A. (2005), «Brand Strategy in Professional Sports: The Case of French Soccer Teams », European Sport Management Quarterly, vol.5, p.23-46.

Dermit-Richard N. (2003), «L'absence chronique de rentabilité financière des clubs de football professionnels: une proposition d'explication », Revue Européenne de Management du Sport, ${ }^{\circ} 9$, avril, p. 1-33.

Di Maggio P. et Powell W. (1983), «The Iron Cage Revisited: Institutional Isomorphism and Collective Rationality in Organizational Fields », American Sociological Review, vol. 48, avril, p. 147-160.

FATF-GAFI (2009), Money Laudering Through the Football Sector, juillet, www.fatf-gafi.org.

DNCG (2011): Rapport d'activité sur les comptes des clubs professionnels, saison 2010/2011. Ligue de Football Professionnel, 103 pages.

Ferreira A. et Otley D. (2009), « The Design and the Use of Performance Management Systems: An Extended framework for Analysis », Management Accounting Research, vol. 20, n4, p. 263-282.

Fontanel G. (2007), Les transformations structurelles des clubs sportifs : recherche d'un cadre d'analyse approprié à leur gestion, Thèse de doctorat en Sciences de Gestion, Université Jean Moulin Lyon 3.

Fréry F. (2009), «Les postures stratégiques face à l'incertitude», 
L'Expansion Management Review, vol. 2, n¹13, p. 20-27.

Gervais M. (2009), Contrôle de gestion, Economica, p. 365-376.

Giard V. et Lagroue P.-Y. (2001), "Contrôle des systèmes productifs travaillant à la commande et soumis à de forts aléas ", ComptabilitéContrôle-Audit, tome 7, vol. 2, p.147-163.

Gouguet J.-J. et Primault D. (2002), «Analyse économique du fonctionnement du marché des transferts dans le football professionnel», Revue des Affaires Européennes, n²-3, p. 305-323.

Gouguet J.-J. et Primault D. (2006), « The French Exception», Journal of Sports Economics, vol. 7, n ${ }^{\circ}$, p. 47-59.

Hasrouri L. (2006), «La dynamique du contrôle : le cas des clubs sportifs professionnels », Cahiers de Recherche du Laboratoire Orléanais de Gestion (LOG), $\mathrm{n}^{\circ} 6,24$ pages.

Helleu B. et Durand C. (2007), «La métropolisation du sport professionnel en Europe et en Amérique du Nord: une approche comparative», M@ppemonde, $\mathrm{n}^{\circ} 110$.

Hoehn T. et Szymanski S. (1999), «The Americanization of European Football», Economic Policy, n 28, avril 1999, p. 205-240.

Kesenne S. (2002), «The Monopsonistic Player Labour Market in a WinMaximising League», European sport management quarterly, n², p. 180187.

Lauzanas J.-M. (2003), «Moyenne entreprise et instrumentation de gestion : le cas des clubs sportifs professionnels », Economies et Sociétés, série "Economie de l'entreprise », vol. 5, n¹3, p. 895-921.

Lechner C. et Gudmundsson S. (2012), «Superior Value Creation in Sports Teams: Resources and Managerial Experience »,M@n@gement, vol. $15, \mathrm{n}^{\circ} 3$, p. 282-312.

Leroux D. (2011), Pratique du commissariat aux comptes dans un club de football professionnel: proposition d'une démarche d'audit, Mémoire d'Expertise Comptable.

Malmi T. et Brown D. (2008), "Management Control Systems as a Package - Opportunities, Challenges and Research Directions », Management Accounting Research, vol. 19, n4, p. 287-300.

Meyssonnier F. (2013), " Nouveaux repères et nouveaux espaces du contrôle de gestion : le cas des activités de service », Congrès de l'AFC 2913 (Montréal), 17 pages.

Paché G. et N'Goala G. (2011), «Les stratégies de création et d'appropriation de la valeur dans un contexte d'incertitude majeure : le cas du football professionnel », Management et Avenir, vol. 6, n 46, p. 53-78.

Pelluault P. (1992), Mise en place des outils de gestion dans un club de 
football professionnel: budgets et tableaux de bord, Mémoire d'Expertise Comptable.

Rousseau O. (2009), L'audit des revenus dans un club de football professionnel: proposition d'une démarche pour le commissaire aux comptes, Mémoire d'Expertise Comptable.

Senaux B. (2004), «Gouvernance des clubs de football professionnels: une approche partenariale », Actes de la $13^{\text {ème }}$ conférence de l'AIMS (Normandie), 27 pages.

Touchais L. (2000), «Sport spectacle et contrôle de gestion : utopie ou réalité ? Étude des clubs de football professionnel», Actes du $21^{\text {ème }}$ congrès de l'AFC, 21 pages.

Touchais L. (2001), «Le contrôle de gestion en situation d'incertitude : le cas du sport spectacle », Finance-Contrôle-Stratégie, vol. 4, n 1 , mars, p. 215-237. 